\title{
Fluid retention syndrome in women
}

\begin{abstract}
The term Fluid Retention Syndrome (abbreviated; FRS) is more accurate than the commonly used term "idiopathic edema "or "cyclical edema ", as it substantiates the view that the disorder has multifactorial etiology. FRS of women may be defined as fluid retention occurring in the absence of well-defined hydrostatic or oncotic mechanism as a result of congestive cardiac failure, hypoproteinemia or local venous or lymphatic obstruction.

FRS may result from humoral, metabolic, autonomic and iatrogenic mechanisms. The syndrome comprises a triad of fluid retention, autonomic disturbance and affective disorder, the latter represents the cornerstone of the syndrome and the condition is a distinctive psychosomatic disorder.

The symptoms of fluid retention are variable and many patients are subjected to multiple hospital referrals for unnecessary investigations, medication or surgery which can be avoided once the unified scope of the syndrome is well appreciated.

From November 1991 - Nov. 1994 we could study 52 cases of typical triad symptoms of FRS between the ages of 16-52 years (mean 36.5 years).

These cases represent our ongoing workup to find out more cases to establish the syndrome as a true and existing entity in our daily medical practice.
\end{abstract}

Volume 4 Issue 5 - 2017

\author{
Saadi JS Alladir \\ Computer science department, Babylon University, Iraq
}

Correspondence: Saadi JS AlJadir, Computer science department, Babylon University, PO Box 498, Nassiriya, Thi Qar, Iraq, Email saljadir5 I@gmail.com

Received: November 29, 2016 | Published: May 17, 2017

\section{Introduction}

\section{Fluid Retention Syndrome in Women (FRS)}

The commonly used term for FRS "Idiopathic edema" is misleading, because sufferers rarely show pitting or dependent edema and contributory risk factors can be defined, the descriptive term "FRS" is more precise as it distinguishes the perspective that the condition has multi- factorial etiology.

The majority of general practitioners think that "FRS" doesn't exist as distinguished condition, but as indefinite or rather nebulous entity associated with women who demand diuretic for "bloating" or "swelling". The condition is frequently, confused with premenstrual tension and some deny its existence dismissing as imaginary the complaints of women who insist that they swell during the day have to spend the evening in loose dressings. ${ }^{1}$

\section{Definition}

"FRS" may be defined as a fluid retention in the absence of welldefined hydrostatic or oncotic mechanism as a result of congestive cardiac failure, hypoproteinemia or local venous or lymphatic obstruction. Most patients are between 20-50 years but the onset may follow menopause. It is rare in children, although the syndrome was found between 2-10 years of age where there is strong family history of the syndrome .FRS is extremely rare in men. ${ }^{1}$

\section{Etiology}

FRS may result from humoral, metabolic, automatic and iatrogenic mechanisms. ${ }^{2}$ Although the definitive etiology is uncertain, increased permeability of capillary walls accentuated by arteriolar vasodilation appears to be a primary culprit in etiology, ${ }^{3,4}$ both mechanisms result in the transfer of fluid from the intravascular to the extravascular compartment with the resulting hypovolemia leading to sodium and water retention through activation of Renin-Angiotensin-Aldosterone system (RAAS).

\section{Clinical Features of FRS}

These comprise a triad of symptoms of fluid retention, autonomic disturbance, and affective disorder. ${ }^{2}$ The second and the third components of the triad are present to a variable degree in the individual patients. Fluid retention leads to complaints of swelling of the face, hands, breasts, abdomen and legs, which becomes worse as the day advances. The patient frequently changes into loose dressings, and the time at which this occurs is a useful assessment of the entity and the severity of this disorder. The patient's face may appear puffy and bloated, rings may be discarded and a distended abdomen may lead to accusations of pregnancy!

Most women retain fluid to some extent, during premenstrual period, and there is no clear dividing line between normal and abnormal fluid retention. Fluid retention is usually accompanied by diurnal weight variation of between 1.4 and $6.0 \mathrm{~kg}$ (3-14lbs.) daily. A diurnal weight variation of more than 1.4 daily is considered abnormal. ${ }^{5}$ Longer term fluctuation weight with a periodicity of 1-2 weeks is not uncommon see Table 1 .

Table I Common symptoms of FRS in Clinical Cases

\begin{tabular}{ll}
\hline Weight gain from fluid retention & Breast tenderness \\
\hline Mood Swing and Irritability or Anger & Tension or Anxiety \\
Appetite Changes and Food Cravings & Depressed Mood \\
Insomnia & Crying Spells \\
Joint or Muscle's Pains & Headaches \\
Abdominal Bloating & Fatigue \\
\hline
\end{tabular}

\section{Parasympathetic over activity}

That includes hyperactivity of the bowel, bladder and vascular system, a history of irritable bowel; with complaint of abdominal pain, intermittent diarrhea and constipation is common and the patients may be referred to gastroenterologist or gynecologist.

Complaints of urge frequency of micturition amounting to urge incontinence and in severe cases may lead to unnecessary antibiotics treatment for urinary "infections" or referral to an urologist.

Intermittent vasovagal attacks, in forms of syncope or dizziness spells are not uncommon and lead to a mistaken diagnosis of epilepsy and neurological referral as well! 
Most fluid retention patients show symptoms of varying degree of affective disorder, this range from minor degree of fatigue, irritability anxiety and depression. Affective symptoms form an integral part of FRS and the condition is a true psychosomatic disorder. ${ }^{6}$

Visual blurring caused by retinal edema is common in fluid retaining patients; and lead to consult the ophthalmologist. Tension headaches commonly accompany affective symptoms, in few patients headaches is severe and worse in the morning resembling headache of increased intracranial pressure, presumably caused by a degree of cerebral edema. Polydipsia result from hypovolemic stimulus following extravasation of fluid from intravascular to extravascular compartment leads to consumption of large quantities of fluid with nocturnal polyuria. If thirst is intense the patient may be suspected of having diabetes mellitus or hysterical water drinking. In summary, the symptoms of FRS are variable and many patients are subjected to multiple hospital referrals for unnecessary investigations, medications or surgery which can be avoided once the scope of the syndrome is appreciated.

\section{Risk factors of FRS}

Recognition of the contributory risk factors in fluid retention is essential for rational and effective management.

\section{Metabolic factors}

Obesity, or a history of weight gain is found in most fluid-retaining patients. A family history of diabetes is common, and acute fluid retention may occur in the both sexes with the institution of insulin therapy for diabetes mellitus ('insulin edema'). ${ }^{7}$ Subacute and chronic forms of diabetic oedema are seen in young women with unstable diabetes. ${ }^{8}$

\section{Endocrine factors}

Thyrotoxicosis, hypothyroidism ${ }^{9}$ and estrogens may be associated with fluid retention. More subtle endocrinopathies may be involved in the etiology of fluid retention. In one study, urinary dopamine levels were low in patients with fluid retention ${ }^{10}$ and, in another, gonadotrophin levels were raised after stimulation with releasing hormones compared with control patients.

\section{Psychiatric factors}

Affective symptoms are common in fluid-retaining patients and onset may follow emotional stress related to life events.

\section{Short-term precipitants}

Prolonged standing, high ambient temperatures, a febrile illness and acute emotional stress, liberal dietary intake (Alcohol, excessive carbohydrate) may accentuate pre-existing fluid retention (Table 2).

\section{Hypothetical Links between the Risk Factors and the Fluid Retention Syndrome}

The nature of the endocrine or metabolic defect responsible for abnormal fluid retention in women is unknown. Increased sympathetic activity may diminish renal sodium and water excretion via diminished renal dopamine synthesis. ${ }^{10}$ Active neurogenic vasodilation with increased bowel and bladder contractility leading to fluid retention, the irritable bowel syndrome and urge frequency of micturition may have a common basis; increased parasympathetic (cholinergic) activity. In fluid-retaining patients, increased autonomic activity appears to be driven centrally via the connections of the hypothalamus with limbic system; the putative site of affective disorder.
Table 2 Major Risk factors for the FRS

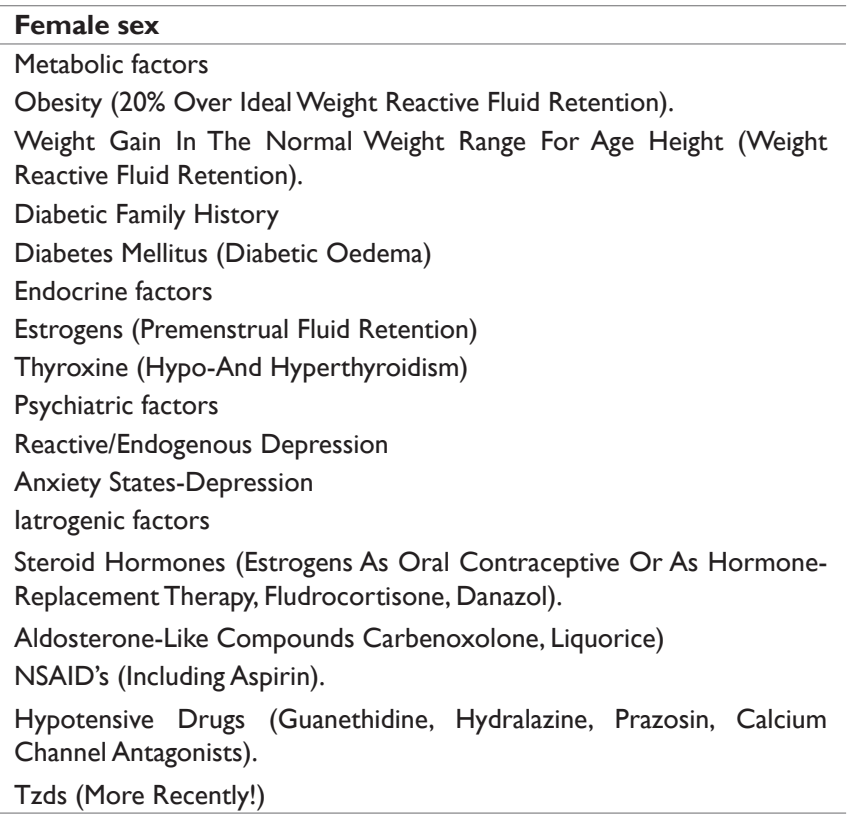

The association of fluid retention with obesity, diabetes mellitus or a diabetic family history may result from the absolute or relative hyperinsulinemia that commonly accompanies these metabolic disorders. Insulin is known to induce renal retention of sodium and water, and insulin-mediated upregulation of cellular sodium/potassium ATPase results in efflux of sodium from the intracellular compartment and fall in intracellular sodium and calcium that will lead to fall in resistance vessel resulting in arteriolar vasodilation increases capillary ultrafiltration and augments fluid retention via arterial hypovolemia. As in T2 diabetes mellitus, insulin responsiveness may be restored by dietary restriction, leading to effective weight loss.

The mechanisms of vasodilating precipitants of fluid retention (such as thyrotoxicosis, fever and high ambient temperature) and of iatrogenic precipitants such as estrogens, danazol and NSAID's are well recognized and require little comment. ${ }^{12,13}$

\section{Management of the fluid retention syndrome}

It is important to reach an accurate diagnosis of the condition based on the exclusion of cardiac, hypoproteinemic and obstructive cases of edema. This may be achieved by appropriate screening investigations, a characteristic history and the demonstration of the abnormal diurnal weight variation by means of weight chart kept by the patient over a period of 2-4 weeks. Most cases respond to abolish or modification of the contributory risk factors (Table 3).

\section{Weight-reactive fluid retention}

Where fluid retention is weight-reactive, the important aspect of management is to return the patient to a target weight. This may be defined either as the patient's weight in early adult life or, less ambitiously, body weight prior to the onset of fluid retention. It is important to set realistic goals for weight reduction. Long-term weight loss of $0.5-1.5 \mathrm{~kg}$ (1-2 lbs.) weekly is satisfactory but losses of up to 2.3-2.7 kg (5-6 lbs.) may occur in the first weeks of dieting as a result of fluid loss. Salt restriction is unnecessary beyond that imposed by reduced caloric intake. Fluid intake should not be restricted.

Symptoms of bloating and swelling subside, accompanying affective symptoms improve, and in many cases, the patient describes 
a sense of well-being and positive health that may have been absent for many years. In most cases of weight-reactive fluid retention, dietary means are sufficient to control symptoms; diuretics are usually unnecessary and not be prescribed initially.

Table 3 Management of the FRS

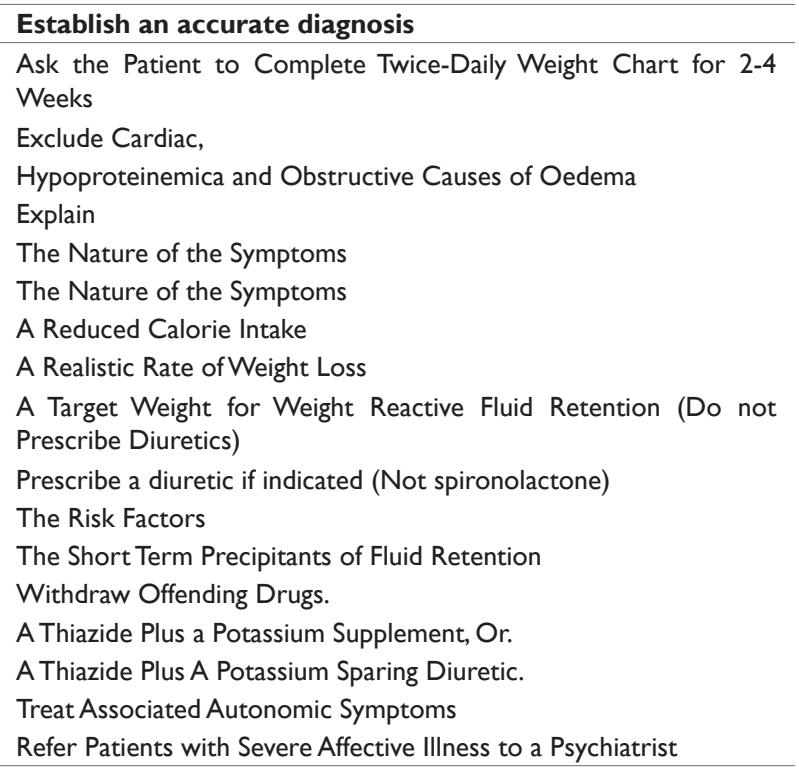

A proportion of non-obese fluid-retaining patients give no history of weight gain before the onset of symptoms. In these patients, the dominant risk factors are usually emotional, and diuretics are effective in controlling symptoms in inverse proportion to the degree of psychiatric abnormality present.

Symptoms of autonomic hyperactivity often improve with treatment of fluid-retaining symptoms by dietary means. Severe symptoms by irritable bowel or urge frequency of micturition may require the prescription of an anticholinergic drug. In patients with severe urge frequency and incontinence of micturition, a referral to urologist for urodynamic studies and bladder retaining capacity may be necessary. Affective symptoms often improve with the treatment of fluid retention, but patients with severe anxiety and depression remain unwell and occasionally require psychiatric referral.

\section{latrogenic fluid Retention}

This ceases when the offending drug is stopped. Fluid retention related to thyroid disorder responds to specific treatment. Diabetic oedema is best managed by dietary measures to achieve ideal weight and the establishment of optimal metabolic control; diuretics may be required in some cases.

\section{Role of diuretics}

The place of diuretics in the management of fluid retention is controversial. On one hand, the only treatment many patients receive is a diuretic prescribed by general practitioner for 'bloating', while on the other, it has been claimed that fluid retention is diuretic-induced illness. ${ }^{15}$

According to the author's experience, fluid-retaining symptoms have invariably preceded the prescription of a diuretic; women are reluctant to take diuretics and anxious to stop them. However, it is possible that diuretics abuse is seen in a few patients referred to specialized centers.
Diuretics are most effective in non-obese fluid retaining women with no history of weight gain or severe affective illness.

As weight increases or psychiatric illness becomes more severe, they become less effective. This may lead to the consumption of increasing doses of diuretic (especially loop diuretics) with the consequent risk of electrolyte depletion. Diuretics are usually ineffective in obese fluid-retaining patients or in patients with severe psychiatric disorder regardless of weight.

A thiazide diuretic with either a potassium supplement or potassium sparing diuretic should be prescribed if required. Loop diuretics should be avoided and spironolactone is no longer recommended for the treatment of fluid retention syndrome.

Many women find that diuretics are required only intermittently to control exacerbation of fluid retention related to the risk factors described above. If these removed or subside spontaneously, diuretics may be gradually reduced and discontinued. However, patients who discontinue diuretics should be warned that withdrawal may be followed by 1-2 weeks of self-limiting rebound oedema.

\section{Role of other drugs}

Several other drugs have been used in the management of the fluid retention in small numbers of patients. These include bromocriptine, levodopa, dexamphetamine, propranolol, captopril and chlorpropamide. Most of clinical expertise sees that these drugs have no place in the management of most patients with fluid retention; their use should be restricted to patients with refractory fluid retention syndrome referred to specialized centers. ${ }^{1}$

\section{Prognosis of the fluid retention syndrome}

The results of managing most patients with weight-reactive fluid retention are good provided that the patient complies with dietary advice and attains target weight. Relapses inevitably occur as a result of the dietary indiscretions when the fluid-retaining symptoms return. These can be controlled by returning to a strict diet and optimum weight.

The prognosis for relief of symptoms is not as good for slim patients with severe affective symptoms related to neurotic or depressive illness or to continuing social stress, or for patients who cannot lose weight. Even in these patients, support and explanation of the nature of the illness is therapeutic, enabling the patient to understand and cope with previously in comprehensible symptoms. The management of this common condition deserves more attention and knowledge of the distinctive scope of the syndrome

\section{Research and Discussion}

I have started conducting this work for the first time in our clinical practice willing for the future of fluid retention clinic to enrich the pool of cases referral.

From autumn 1991 to November 1994 I could register 52 women of typical triad symptoms of the syndrome, between the age of 16 to 52 years (mean age is 36.5 years) and for ethical purpose I asked our registrars in the medical unit in Merjan Teaching Hospital, Babylon for case reporting in specific printed formula.

We have met a lot of technical, laboratory and economic difficulties and lack of endocrine assays because of the present situation of our country due to imposed sanction, but we could overcome those problems by extensive medical efforts, utilizing the available facilities coupled with intensive follow up of cases. 
We have study those cases by screening them with the appropriate investigations to exclude other causes of oedema. We informed the patients to have weight chart (2-4 weeks), but for economic reasons we have received only 20 weight charts that eventually showed the diurnal variation in body weight.

We could not register children cases although pediatricians have mentioned undefined oedema in a few children. Strong family history of the syndrome could be obtained in majority of patients. History of diabetes mellitus and diabetic family and thyroid disease were present in significant number of cases $^{24,14}$ respectively and in $^{14}$ multiple endocrine disorders (Diabetes mellitus, thyroid disease, menstrual disorders...) could be observed. Over weight was found in most patients and psychiatric patients with ideal body weight was detected in lesser number of cases.

We will continue our ongoing work up to find our more cases to establish the syndrome as existing true entity in our current medical practice.

\section{Acknowledgements}

I would like to conduct my thanks and appreciation to Dr. A.S. Jaber, Dr. R.J. Al-Janabi (Merjan Hospital), Dr. Ali Younis MRCPsych, for Psychiatric assessments and Mr. T.A. Al-Assadi for the great help in preparing the computer manuscript (Computer science department, Babylon University).

\section{Conflicts of interest}

The author declares there is no conflict of interest.

\section{References}

1. Dunnigan MG. Management of the fluid retention syndrome in women, Hospital Update. UK, 1990, pp. 653-663.

2. Dunnigan MG. Recognition and management of the fluid retention (idiopathic or cyclical oedema) and premenstrual syndromes. In: McNaughton MC (Ed.), Medical gynecology, Blackwell scientific, Edinburgh, UK, 1985 ; p. 27-55.
3. Streeten DPH. Idiopathic oedema: pathogenesis, clinical features and treatment. Metabolism. 1987;27(3):253-383.

4. Edwards OM, Bayliss RI. Idiopathic oedema of women. A clinical and investigative study. $Q J$ Med. 1976;(177):125-144.

5. Thorn GW. Approach to the patient with idiopathic oedema or periodic swelling. JAMA. 1986;206(2):333-338.

6. Pelosi AJ, Sykes RA, Lough JR, et al. Psychiatric abnormalities in idiopathic oedema. Lancet. 19886;2(8514):999-1002.

7. Sims EA, MacKay BR, Shirai T. The relationship of capillary angiopathy and diabetes mellitus to idiopathic oedema. Ann intern Med. 1965;63(6):972-987.

8. Dunnigan MG. Unusual manifestations of diabetes mellitus. Practitioner. 1979;222(1329):321-330.

9. Al-Kader AA, Aber GM. The relationship between the idiopathic oedema: syndrome and subclinical hypothyroidism. Clin Endocrinol. 1979;10(3):271-279.

10. Kuchel O Cuche JL, Buu NT, et al. Catecholamine excretion in idiopathic oedema: decreased dopamine exertion a pathogenic factor. $J$ Clin Endocrinol Metab. 1977;44:39-646.

11. Young JB, Brownjohn AM, Chapman C, et al. Evidence for a hypothalamic disturbance in cyclical oedema. Br Med J. 1983;286(6379):16911693.

12. Greenfield ANM. Survey of the evidence for active neurogenic vasodilation in man. Fed Peoc. 1966;25(6):1607-1610.

13. Williams RB. Textbook of endocrinology. WB Saunders, London, England, UK, 1974; pp. 521-527.

14. MacGregor GA, Markandu ND, Roulston JE, et al. Is idiopathic oedema idiopathic? Lancet. 1979;1(8113):397-400. 